\title{
NOREPINEPHRINE-CONTAINING LOCUS COERULEUS NEURONS IN BEHAVING RATS EXHIBIT PRONOUNCED RESPONSES TO NON-NOXIOUS ENVIRONMENTAL STIMULI ${ }^{1}$
}

\author{
G. ASTON-JONES*,2 AND F. E. BLOOM
}

The Salk Institute, San Diego, California 92138 and ${ }^{*}$ California Institute of Technology, Pasadena, California 91125

\begin{abstract}
The effect of sensory stimulation on discharge in norepinephrine-containing locus coeruleus (NE-LC) neurons was studied in unanesthetized behaving rats. Single unit and multiple unit extracellular recordings demonstrated consistent patterns of response. (1) Short latency (15- to 50-msec), transient, biphasic changes in discharge were elicited predictably by non-noxious auditory, visual, and somatosensory stimuli; individual recordings typically exhibited polysensory responsiveness. (2) Sensory-evoked field potentials (FPs) were synchronized with unit responses simultaneously recorded from the same electrodes. (3). The magnitudes of sensory-evoked response varied as a function of vigilance, so that the largest responses occurred for stimuli which awakened animals and the least responsiveness was exhibited during uninterrupted sleep. (4) Sensory responsiveness decreased during grooming and sweet water consumption, similar to the results for sleep. (5) Characteristic response properties were topographically homogeneous throughout the NE-LC. (6) Discharge was synchronized markedly among neurons in multiple unit populations during phasic robust responses.

These results are interpreted in light of the preceding report (Aston-Jones, G., and F. E. Bloom (1981) J. Neurosci. 1: 876-886) and studies of the postsynaptic effects of NE to indicate that the NE-LC system may function more in phasic processes than in modulation of the tonic arousal level. We propose that pronounced NE-LC discharge may enhance activity within target cell systems primarily concerned with processing salient external stimuli and suppress central nervous system activity related more to tonic, vegetative functions. Thus, the NE-LC system may bias global behavioral orientation between stimuli in the external versus internal environments.
\end{abstract}

Most brain norepinephrine (NE)-containing neurons are located in the pontine nucleus locus coeruleus (LC). The extensive projections of these neurons, together with the pronounced behavioral, clinical, and postsynaptic effects produced by manipulations of $\mathrm{NE}$ activity in brain, have fostered several possible functions for the NE-containing LC (NE-LC) system (Foote et al., 1975; Segal and Bloom, 1976a, b; Freedman et al., 1977). Depending upon the lability of NE-LC activity, previous data could support the view that this system regulates either long duration brain activity, such as sleep and

\footnotetext{
${ }^{1}$ This work was supported by United States Public Health Service Grant AA 03504, National Institutes of Health Training Grant GM 02031, and National Institutes of Health Grant NS 16209. We thank Dr. Steve Foote for advice throughout, Ms. S. Aston for help in data analysis, and Ms. N. Callahan for typing the manuscript. This paper was submitted by G. A. -J. in partial fulfillment of the requirements for the degree of Doctor of Philosophy, California Institute of Technology.

${ }^{2}$ To whom correspondence should be addressed at his current address: The Arthur V. Davis Center for Behavioral Neurobiology, The Salk Institute, P. O. Box 85800, San Diego, CA 92138.
}

waking, or short duration events, such as phasic attention and distractibility. Other studies (Graham and Aghajanian, 1971; Bunney et al., 1975; Cedarbaum and Aghajanian, 1976; Aghajanian et al., 1977; Bird and Kuhar, 1977; Aghajanian, 1978; Aston-Jones et al., 1980) have reported consistently that NE-LC neurons in anesthetized preparations spontaneously discharge in a slow tonic manner and are apparently responsive only to strongly noxious stimuli. Such tonic discharge, insensitive to nearly all environmental stimuli, is consistent with previous hypotheses linking this system with stages of the sleepwaking cycle (Ramm, 1979; Clark, 1979; Amaral and Sinnamon, 1977; Steriade and Hobson, 1976). On the other hand, some investigators (Lader, 1974; Gray et al., 1975; Redmond and Huang, 1979) have proposed that predominant responsivity to noxious stimuli indicates a role for the NE-LC system in mediating pain and anxiety.

We have found, however, that, in unanesthetized behaving animals, the discharge of NE-LC neurons is much more labile than that in anesthetized preparations. In the preceding paper (Aston-Jones and Bloom, 1981), we reported that spontaneous activity varies not only with 
tonic stages of the sleep-waking cycle (S-WC) but also with phasic EEG events within some stages (spindle activity during slow wave sleep). Furthermore, we have demonstrated (Jones et al., 1979; Foote et al., 1980) that NE-LC neurons in behaving rats and monkeys are responsive to mild, non-noxious environmental stimuli of many modalities. In addition, other laboratories (Aghajanian et al., 1977; Takigawa and Mogenson, 1977) have reported similar responses to electrical stimulation of certain peripheral nerves. These various data indicate that the NE-LC system may function principally in more phasic processes than sleep and waking and in phenomena more general than pain and anxiety.

We therefore undertook a detailed analysis of NE-LC sensory responsiveness in unanesthetized behaving rats and now report characteristics not previously described. (1) The magnitudes of response elicited by environmental stimuli vary as a function of vigilance levels. (2) Sensory-elicited discharge is accompanied synchronously by evoked field potentials from the same electrodes. (3) Sensory-evoked discharge decreases within active waking during grooming and consumption.

We conclude that the NE-LC system may play a specific role within the general framework of arousal, altering central nervous system (CNS) and behavioral responsivity to salient unexpected stimuli in the external environment.

\section{Materials and Methods}

Surgery. Rats were prepared for experimental recording sessions as previously described (Aston-Jones and Bloom, 1981). In brief, the LC was localized during stereotaxic surgery with the aid of unit recording. Skull screws were implanted to provide electroencephalogram (EEG) and ground reference signals, and bilateral subcutaneous wires were sutured permanently through dorsal neck muscles to serve as electromyogram (EMG) electrodes.

Experimental paradigm. After at least 4 days of recovery from surgery, rats were placed in the experimental chamber either as freely moving $(N=82)$ or as harnessrestrained subjects $(N=35)$ (Aston-Jones and Bloom, 1981). For each rat, the experimental paradigm alternated between recording spontaneous activity during natural sleep and waking (Aston-Jones and Bloom, 1981) and recording activity during systematic presentation of sensory stimuli (reported here). The investigator monitored all data collection.

Recording techniques. Filtered and unfiltered unit electrode traces, EEG, EMG, and digital impulse records were obtained as described (Aston-Jones and Bloom, 1981). These signals, along with digital logic pulses synchronized with experimental stimuli (sync pulses), were recorded on magnetic tape and also were displayed on polygraph paper either on- or off-line. Sync pulses and digitized unit activity were fed into a PDP 11-10 or 11-03 computer either on- or off-line to generale peri-stimulus time histograms (PSTHs); inter-spike interval histograms (ISHs) were generated similarly from spontaneous discharge.

Experimental sensory stimuli. The following stimuli were presented at regular 4- or 16-sec intervals in blocks of 25 to 100 trials per modality: (1) tone pips $(4 \mathrm{KHz}, 20$ msec duration, about $96 \mathrm{~dB}$ on a $57-\mathrm{dB}$ background); (2) light flashes (10 $\mu \mathrm{sec}$ duration, 50 to 100 candela); (3) brief, mild skin contacts (touches, manually applied to dorsorostral tail surface); (4) single droplets of a $5 \%$ aqueous glucose solution (dispensed from a remote, electrically activated solenoid); sync pulses for licks were generated by a high impedance circuit upon tongue contact with the solution. Digital sync pulses were synchronized precisely with tone pips, flashes, and licks; a manual push-button generated digital pulses approximately synchronized with touches.

Localization of recording sites. Histological procedures were described previously (Aston-Jones and Bloom, 1981). All data were obtained from histologically identified recording sites, using gliotic lesion sites or Prussian Blue-reacted iron deposits (illustrated in Fig. 1) for reference marks.

Data analysis. Criteria for acceptable single unit (SU), multiple unit (MU), and field potential (FP) data and for scoring the S-WC are described elsewhere (AstonJones and Bloom, 1981). Waking (W) consisted of at least 3 sec of uninterrupted stage I (SI) or stage II (SII), and slow wave sleep (SWS) contained at least 3 sec of uninterrupted stage III (SIII) or stage IV (SIV) (see AstonJones and Bloom, 1981 for a description of the stages).

For SU recordings, each stimulus trial was categorized as to EEG arousal levels which occurred before and after the stimulus: (1) SWS/W, SWS preceded the stimulus which was followed within 0.5 sec by W; (2) W/W, continuous $\mathrm{W}$ both before and after the stimulus; (3) SWS/SWS, continuous SWS both before and after the stimulus. Trials that could not be assigned to one of these categories unambiguously were not included in the data analysis. The magnitude of response for each stimulus trial was determined (from polygraph records) by subtracting the number of impulses in the $200-\mathrm{msec}$ epoch preceding the stimulus from the number of impulses in the 200-msec epoch following the same stimulus.

Response latencies were determined by computer from PSTHs which incorporated at least 25 consecutive trials each as illustrated in Figure 2. Base line mean and standard deviation (SD) for PSTH hins (bin width $=4$ or $8 \mathrm{msec}$ ) were calculated for a period of $1 \mathrm{sec}$ or more, beginning at least $2 \mathrm{sec}$ after stimulus onsets. Excitatory response onsets and inhibitory response offsets were defined at the midpoint of the first bin which exceeded base line mean by 2 SDs or more and which also was the first of 5 consecutive bins whose mean value met the same criterion. Inhibitory response onsets and excitatory response offsets were defined similarly but using 20 consecutive bins; for inhibitory responses only, if the base line mean was less than 2 SDs, responses were defined using a $1-\mathrm{SD}$ requirement.

\section{Results}

Spontaneous discharge. In general, SU recordings in the NE-LC yielded slow, tonic spontaneous discharge (W rate $=1.74 \pm 0.15 \mathrm{~Hz}$; mean $\pm \mathrm{SEM}, N=30$ cells). However, activity was altered dramatically by mild sensory stimuli. This effect was most obvious in MU recordings, which typically contained aperiodic impulse bursts, each followed by a prolonged period of suppressed activ- 


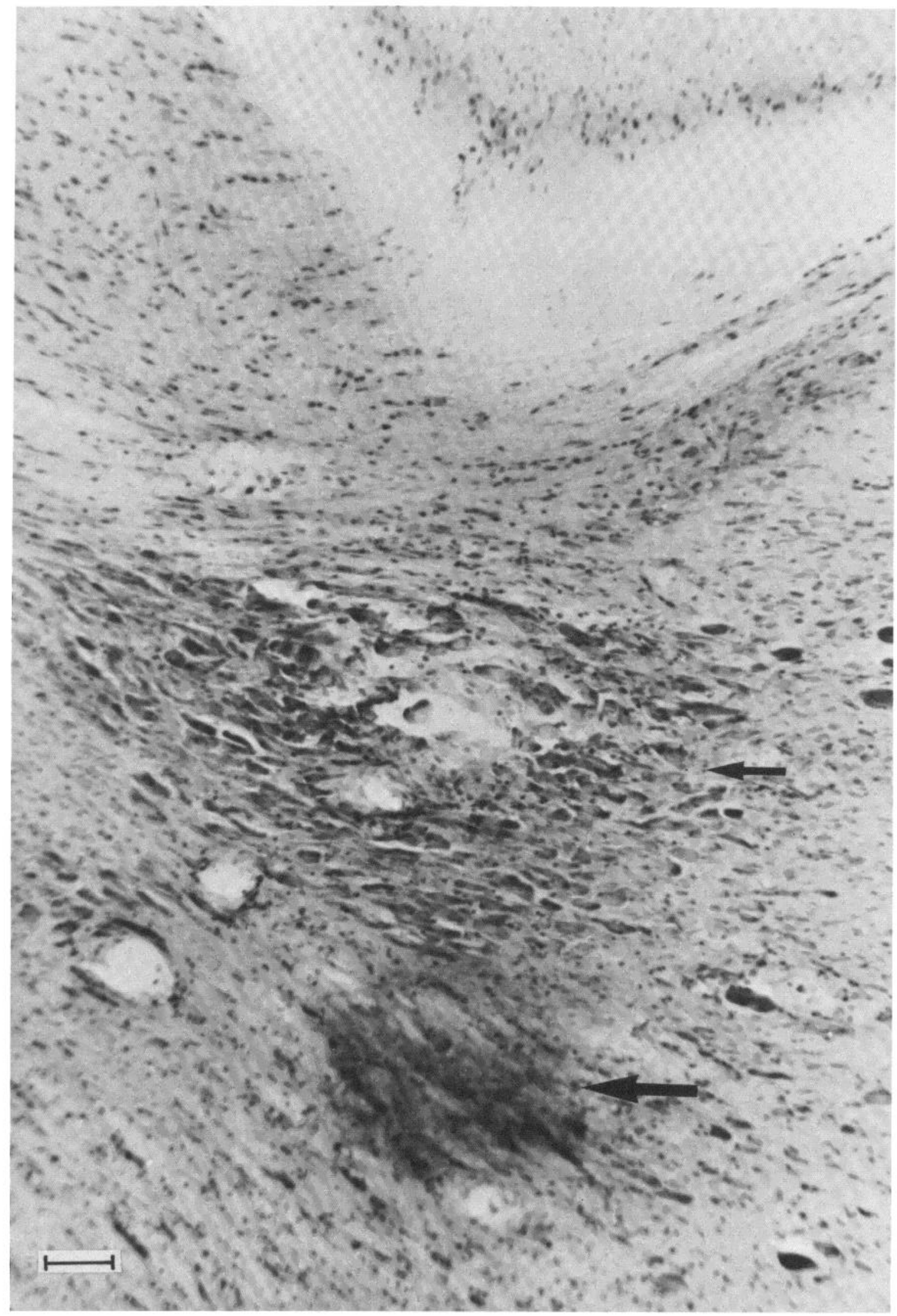

Figure 1. Histological localization of recording sites. A sagittal 40 - $\mu$ m-thick section through the LC (small arrow) from experimental rat brain stained with neutral red is shown. Prussian Blue spot (large arrow) marks iron deposited by recording electrode $100 \mu \mathrm{m}$ ventral to typical LC activity. Calibration bar $=100 \mu \mathrm{m}$. 


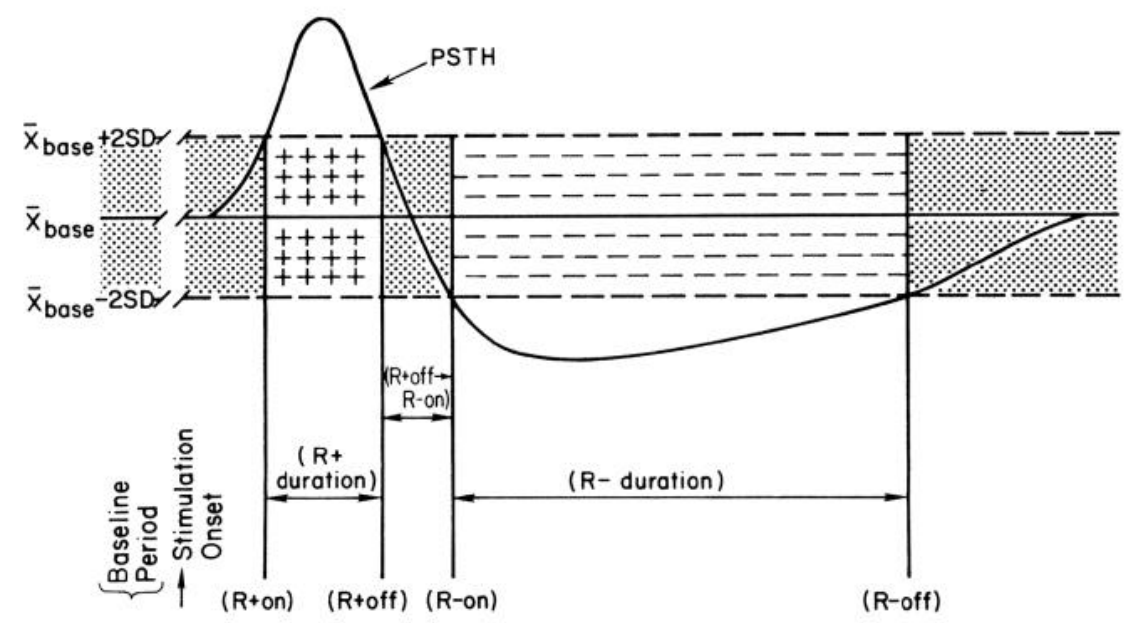

Figure 2. Method used to calculate unit response latencies. Latencies of excitatory response $(R+o n$ and $R+o f f)$ and of inhibitory response $(R-o n$ and $R$-off) were taken at PSTH bins differing from the base line mean $\left(\bar{X}_{\text {base }}\right)$ by predetermined standard deviation $(S D)$ criteria as described under "Materials and Methods." Areas filled with dots and plus and minus signs indicate nonsignificant, excitatory, and inhibitory response periods, respectively. Response durations were defined between $R$ on and $R$ off.
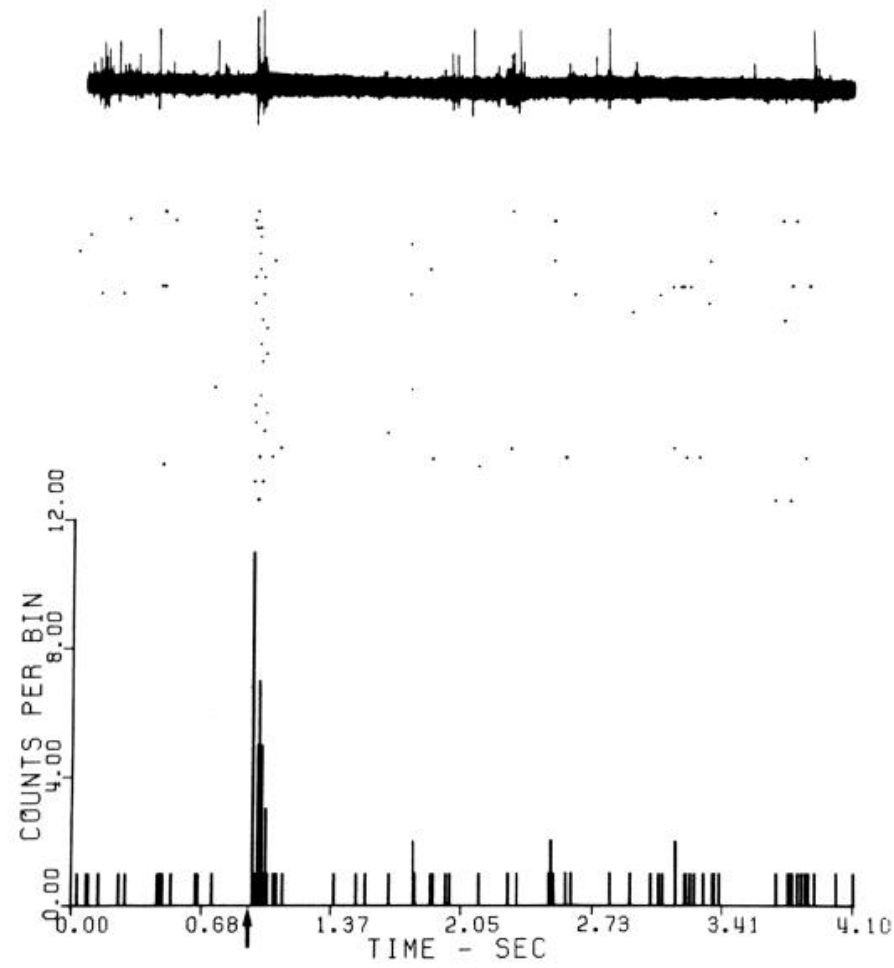

Figure 3. Tone pip-evoked discharge in a single NE-LC neuron. Upper panel, Single oscilloscope sweep of analog discharge trace for one trial. Dots were generated for spikes meeting waveform discriminator criteria. Middle panel, Raster display of impulse activity for 40 consecutive trials, in sequence from top to bottom. Lower panel, PSTH accumulated for 50 consecutive trials (bin width $=8 \mathrm{msec}$ ). Time axis and tone pip onsets (arrow) apply to all panels. ISH and other sensory modality PSTHs for this neuron are given in Figure 4.

ity. These phasic bursts were associated consistently with background stimuli in the environment. Upon systematic examination, we found that this discharge pattern matched sensory-evoked activity characteristic of both MU and SU recordings (described below).

Sensory-evoked discharge. As illustrated in Figures 3 through 6 and summarized in Table I, NE-LC neurons homogeneously responded to mild, non-noxious sensory stimuli of many modalities. Auditory, visual, and somatosensory stimuli all elicited similar patterns of biphasic response, consisting of an initial burst of impulses followed immediately by a longer period of decreased activity (closely resembling the phasic pattern of "spontaneous" activity in MU recordings described above).

Table II summarizes response latencies for tone pip and flash stimuli. MU recordings consistently yielded less variable latencies and fewer response failures than SU data. The response properties of NE-LC neurons in MU populations were also predominantly homogeneous (see Table I and below) as reflected in the markedly synchronized response activity among neighboring NELC neurons recorded simultaneously. MU activity, therefore, may provide more representative latency data than SU recordings, owing to the correspondingly larger sample of impulses. MU response latencies for tone pip and flash stimuli are compared graphically in Figure 7. Excitatory responses to flashes had longer onset latencies than did responses to tone pips (for SUs and MUs, $p<0.0005$ by paired $t$ tests, $N=10$ and 9 , respectively). The same relationship existed for excitation offset latencies in these two modalities $(p<0.005)$, as well as for the subsequent latencies of inhibitory response onset (for SUs and MUs, $p<0.05$ by paired $t$ tests, $N=4$ and 7 , respectively). Excitatory response durations were longer for flashes in SU data $(p<0.005$ by paired $t$ test) but did not differ significantly in MU recordings ( $p>0.1$ by paired $t$ test); the difference here between SU and MU data may be a 

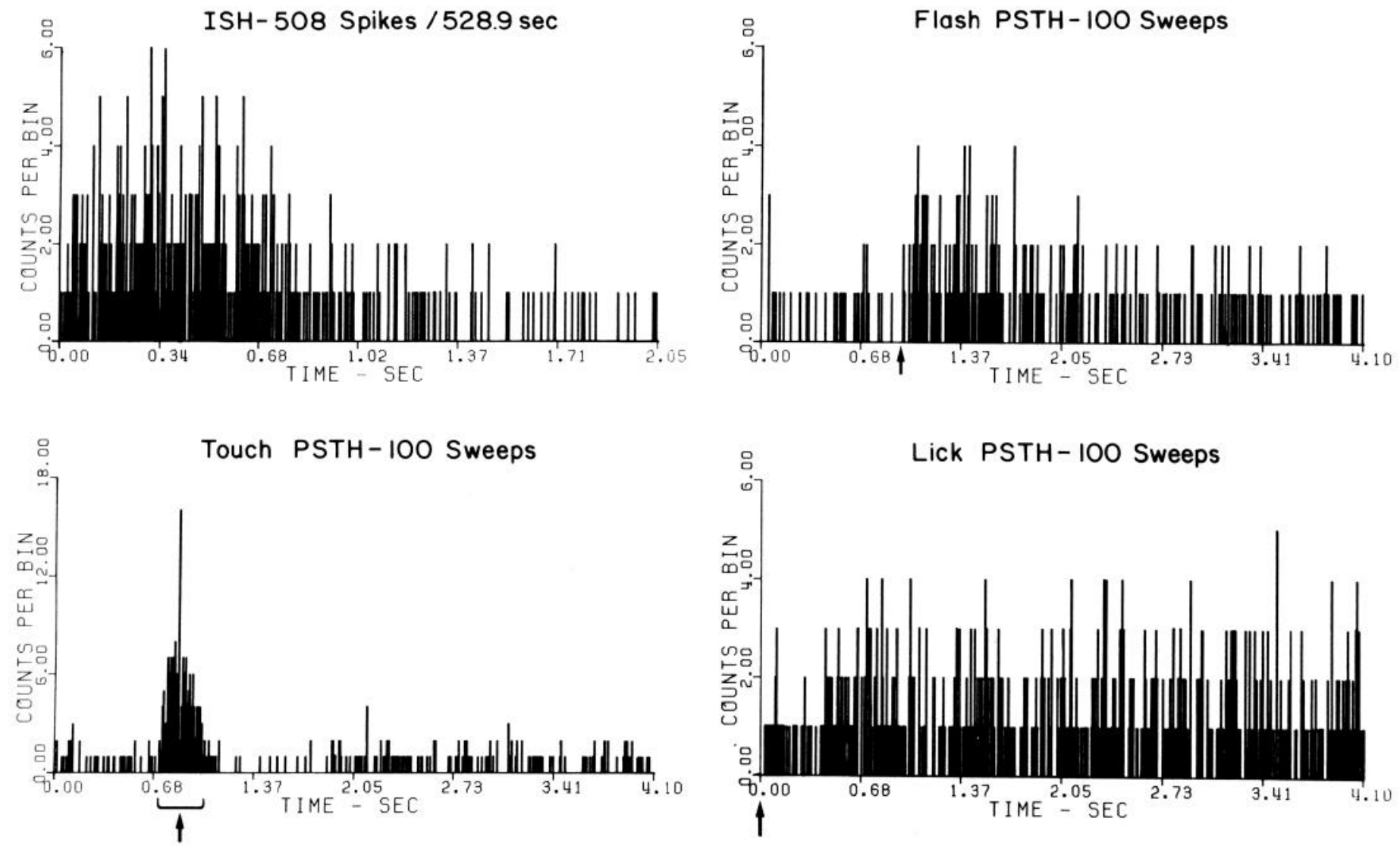

Figure 4. Spontaneous and sensory-evoked discharge in a single NE-LC neuron. The same neuron is shown in all of the panels. The tone pip data for this neuron are given in Figure 3. Arrows indicate stimulus onsets.
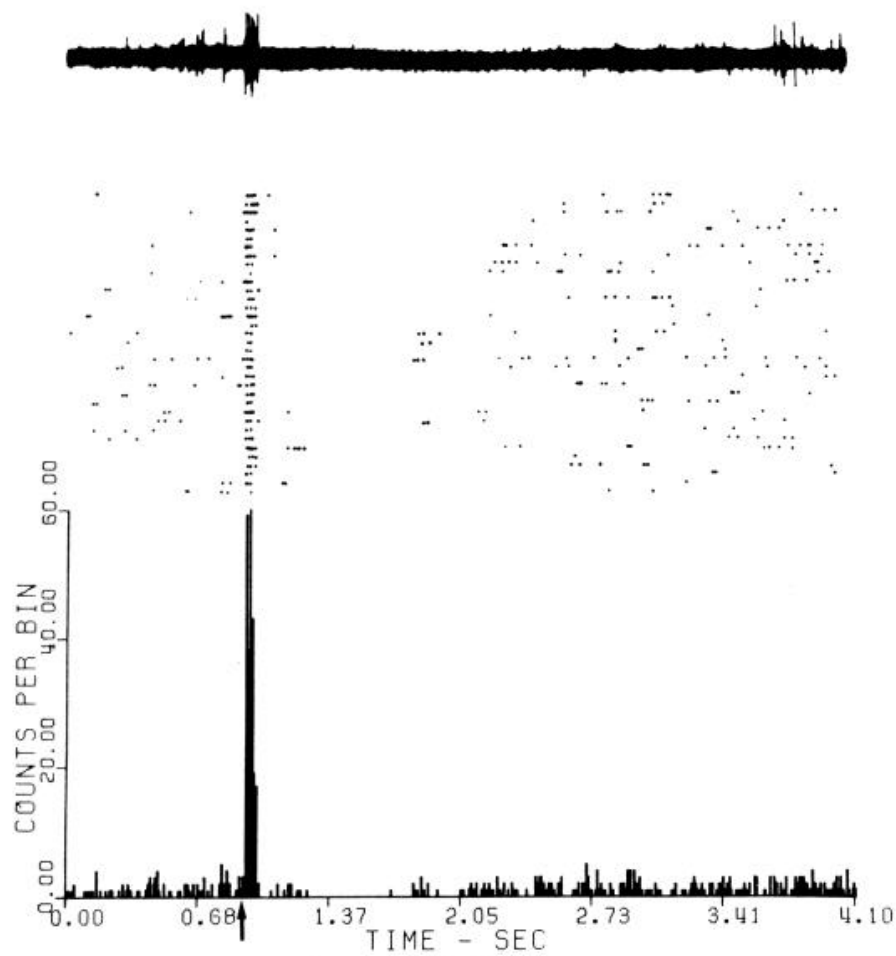

Figure 5. Tone pip-evoked discharge in an NE-LC MU recording. Panel format, time scale, and stimuli are as in Figure 3. ISH and other sensory modality PSTHs for this recording are given in Figure 6. consequence of the relatively high variability in SU data due to the intrinsically small sample of impulse activity as noted above. Inhibitory response durations for tone pips were longer than for flashes (for SUs, $p<0.01, N$ $=4$; for MUs, $p<0.025, N=7$, paired $t$ tests), but the latencies of inhibitory response offset were similar for the two modalities in both SU and MU recordings ( $p>$ 0.1 by paired $t$ tests).

Six SU and three MU touch PSTHs were analyzed quantitatively; although sync pulses were not synchronized precisely with somatosensory stimuli, consistent response patterns were approximated; SU excitatory response duration $=206.6 \pm 40.9 \mathrm{msec}($ mean \pm SEM $)$ and subsequent inhibitory response duration $=532.0 \pm 58.0$ msec; $\mathrm{MU}$ excitatory response duration $=240.0 \pm 24.0$ msec and subsequent inhibitory response duration = $605.3 \pm 127.2$ msec. Touches apparently elicited more pronounced responses than tone pips or flashes. Similarly, auditory responses were generally more robust than responses to visual stimuli, consistent with the comparatively large number of visual response failures (seen in Tables I and II) and flash responses insufficient in magnitude to permit single trial analysis (see below). It was also apparent that touches elicited greater orienting response than tone pips which, in turn, were more effective than flashes. Thus, stimulus modalities yielded the same order of efficacy for NE-LC unit response and for behavioral orienting responses. This indicates that NE-LC responsiveness may vary with evoked vigilance increase more directly than with stimulus modalities per se.

Several NE-LC recordings were examined with olfac- 

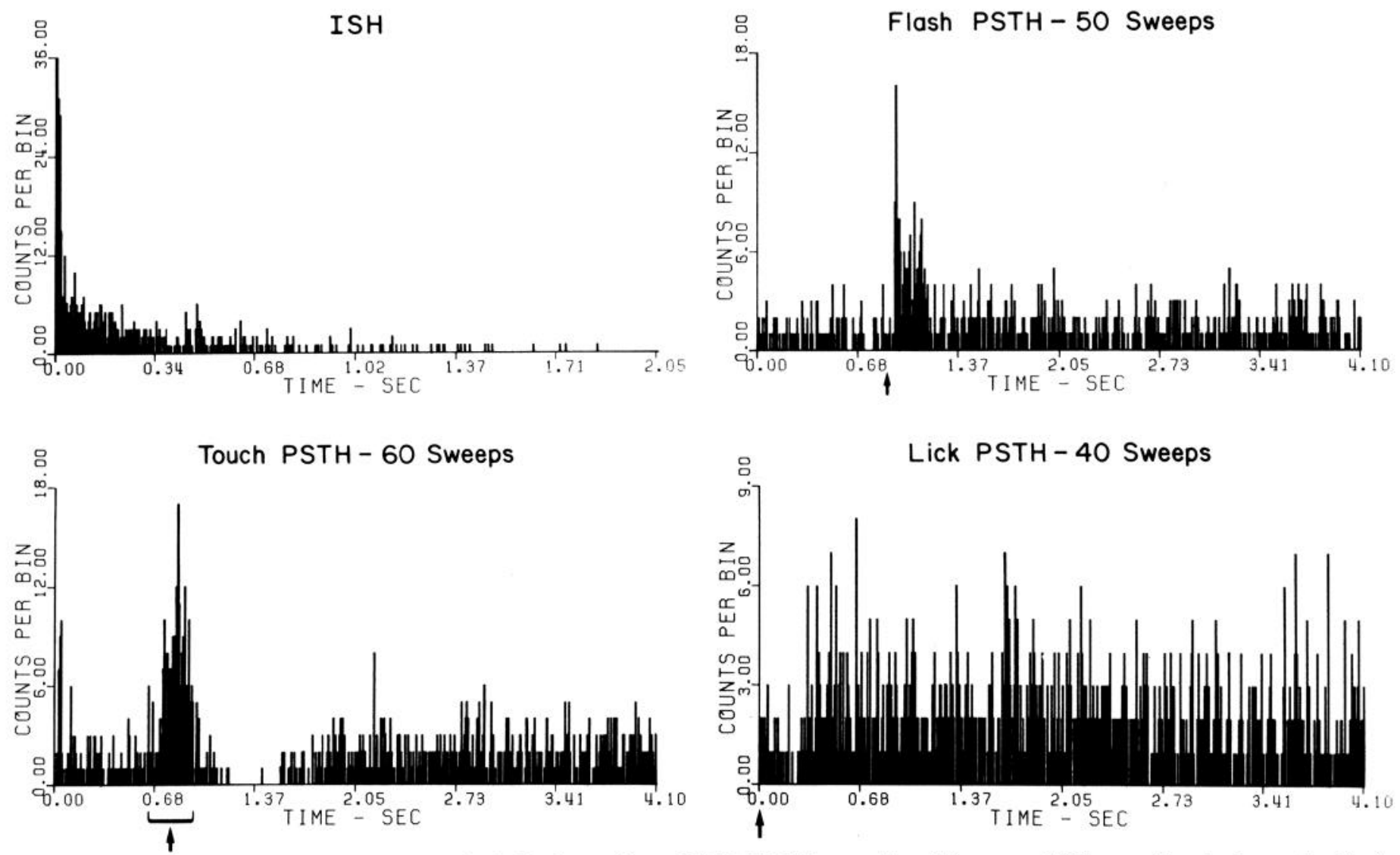

Figure 6. Spontaneous and sensory-evoked discharge in an NE-LC MU recording. The same MU recording is shown in all of the panels. The tone pip data for this recording are given in Figure 5. Arrows indicate stimulus onsets.

TABLE I

Proportions of NE-LC recordings exhibiting characteristic discharge properties

The number of recordings exhibiting each column property over the number of recordings examined. The properties in the columns apply to $\mathrm{SU}$ and MU recordings, except for spontaneous discharge, as listed. Plus (+) and minus (-) signs indicate excitatory and inhibitory responses, respectively.

\begin{tabular}{|c|c|c|c|c|c|}
\hline $\begin{array}{c}\text { LC } \\
\text { Recording }\end{array}$ & $\begin{array}{c}\text { Slow, Tonic } \\
\text { Spontaneous Discharge }\end{array}$ & $\begin{array}{l}\text { Initial + Auditory } \\
\text { Response }\end{array}$ & $\begin{array}{l}\text { Initial + Visual } \\
\text { Response }\end{array}$ & $\begin{array}{l}\text { Initial + Touch } \\
\text { Response }\end{array}$ & $\begin{array}{c}\text { Biphasic }(+,-) \\
\text { Responses in at } \\
\text { least Two Modalities }\end{array}$ \\
\hline \multirow[t]{2}{*}{ SU } & $35 / 42$ & $37 / 38$ & $22 / 27$ & $24 / 24$ & $29 / 30$ \\
\hline & $\begin{array}{c}\text { Phasic, Bursty } \\
\text { Spontaneous Discharge } \\
\end{array}$ & & & & \\
\hline MU & $52 / 53$ & $51 / 51$ & $42 / 43$ & $42 / 42$ & $46 / 47$ \\
\hline
\end{tabular}

TABLE II

Latencies for sensory-evoked responses in NE.LC discharge

Latencies (mean \pm SEM) were determined from PSTH analysis using the 2 SD criterion or 1 SD criterion (see "Materials and Methods"); note that some flash PSTHs did not meet the response criteria.

\begin{tabular}{|c|c|c|c|c|c|c|c|}
\hline \multirow{2}{*}{ Stimulation } & \multirow{2}{*}{ Recording } & \multicolumn{2}{|c|}{ Excitation } & & \multicolumn{2}{|c|}{ Inhibition } & \\
\hline & & Onset & Offset & & Onset & Offset & \\
\hline & & \multicolumn{3}{|c|}{ msec } & \multicolumn{2}{|c|}{ msec } & \\
\hline \multirow[t]{2}{*}{ Tone pip } & SU & $18.0 \pm 1.0$ & $96.6 \pm 5.4$ & $\left(N=17^{a}\right)$ & $232.8 \pm 54.2$ & $610.1 \pm 66.5$ & $\left(N=17: 9^{a}+8^{b}\right)$ \\
\hline & MU & $16.9 \pm 0.7$ & $97.6 \pm 5.5$ & $\left(N=15^{a}\right)$ & $122.1 \pm 10.5$ & $760.9 \pm 74.9$ & $\left(N=15: 12^{a}+3^{b}\right)$ \\
\hline \multirow[t]{2}{*}{ Flash } & SU & $70.8 \pm 9.0$ & $324.1 \pm 52.5$ & $\begin{aligned}(N & =10^{a} \\
N & \left.=1 \mathrm{NS}^{c}\right)\end{aligned}$ & $961.5 \pm 143.6$ & $1141.5 \pm 130.2$ & $\begin{aligned}(N & =4: 3^{a}+1^{b} \\
N & \left.=7 \mathrm{NS}^{c}\right)\end{aligned}$ \\
\hline & MU & $52.0 \pm 3.7$ & $136.0 \pm 9.9$ & $\left(N=7^{a}\right)$ & $329.4 \pm 68.4$ & $548.6 \pm 75.6$ & $\begin{aligned}(N & =7: 6^{a}+1^{b} \\
N & \left.=5 \mathrm{NS}^{c}\right)\end{aligned}$ \\
\hline
\end{tabular}

${ }^{a}$ Data based on 2 SD criterion.

${ }^{b}$ Data based on 1 SD criterion.

${ }^{c}$ NS, no significant response. 


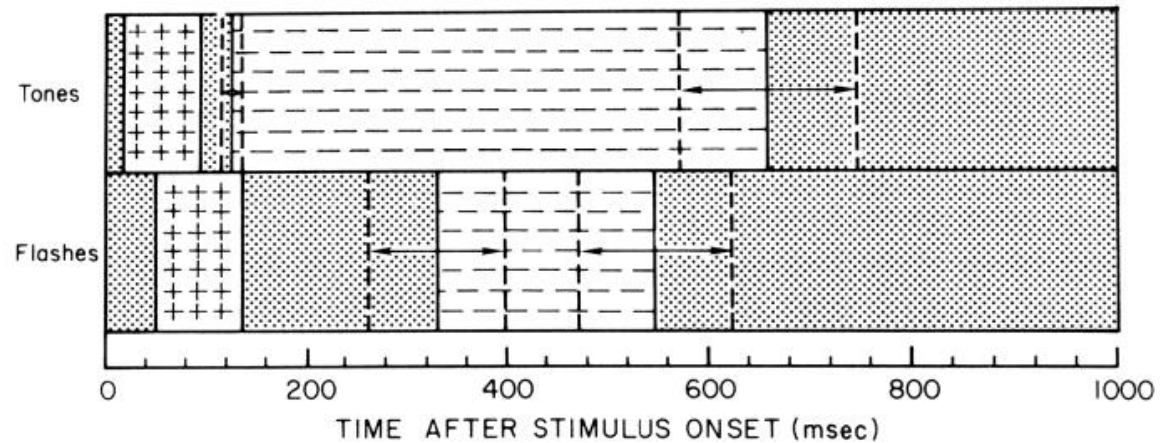

Figure. 7. Latencies of evoked response in NE-LC discharge for tone pip versus flash stimuli. The data are averaged for one group of $7 \mathrm{MU}$ recordings tested with both tone pip (T) and flash (F) stimuli. Solid and adjacent dashed vertical lines indicate mean and SEM latencies, respectively. Areas filled with dots and plus and minus signs indicate nonsignificant, excitatory, and inhibitory response periods, respectively. T+on $<\mathrm{F}+$ on $(p<0.0005)$; $\mathrm{T}+$ off $<\mathrm{F}+$ off $(p<0.005)$; $\mathrm{T}+$ dur $\cong \mathrm{F}+$ dur $(p>0.1) ; \mathrm{T}-$ on $<\mathrm{F}-$ on $(p<0.05) ; \mathrm{T}-$ off $\cong \mathrm{F}-$ off $(p>0.1) ;$ $\mathrm{T}$-dur $>\mathrm{F}-$ dur $(p<0.05) ; \mathrm{T}-$ on minus $\mathrm{T}+$ off $<\mathrm{F}$ - on minus $\mathrm{F}+$ off $(p<0.05)$, where $\mathrm{T}+\mathrm{on}, \mathrm{T}+\mathrm{off}, \mathrm{F}+\mathrm{on}$, and $\mathrm{F}+$ off are excitatory response latencies and $\mathrm{T}-\mathrm{on}$, $\mathrm{T}-$ off, $\mathrm{F}$-on, and $\mathrm{F}$-off are inhibitory response latencies; dur refers to response duration. Paired $t$ tests were used.

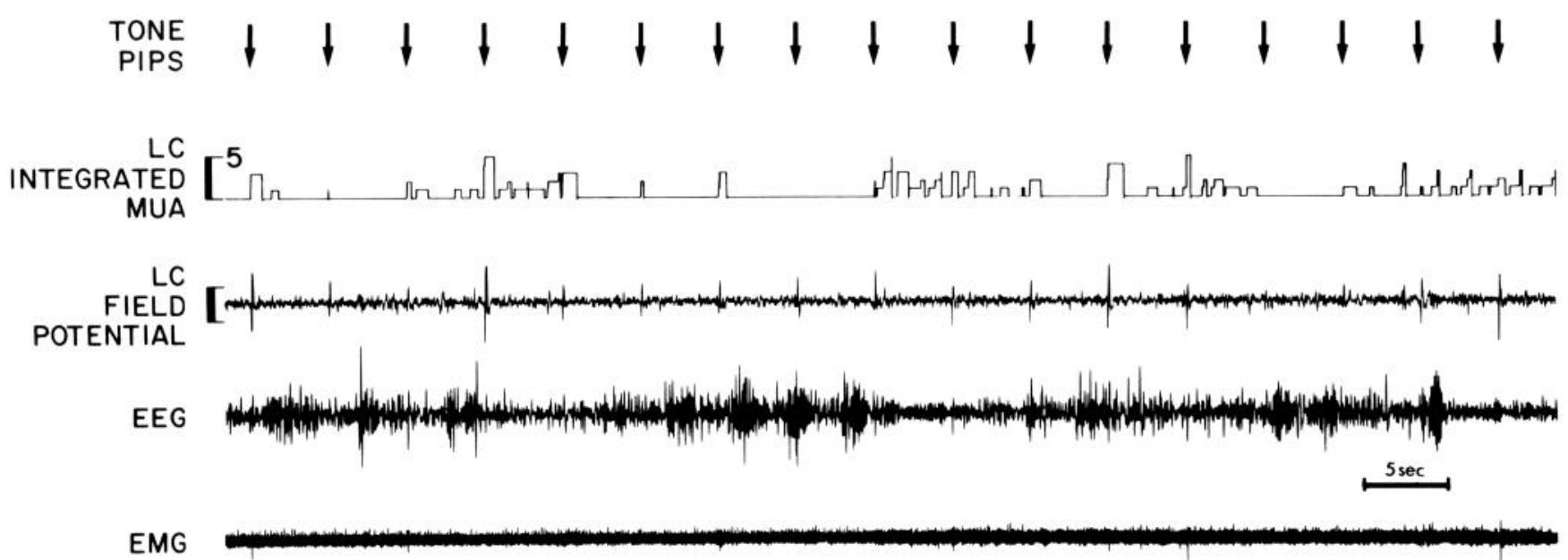

Figure 8. Sensory-evoked FPs and unit responses in the NE-LC during fluctuating cortical arousal. Tone pips (at arrows) evoke smaller magnitudes of MU response during SWS (high amplitude, low frequency, periodic EEG) than during W (low amplitude, aperiodic EEG). The largest amplitudes occur for stimuli that evoke abrupt W from SWS. Differential recordings between adjacent microwires are separated into FP and discharge traces as described under "Materials and Methods." FP calibration $=100 \mu \mathrm{V} . M U A$, MU activity.

tory stimuli (acetic acid-soaked swab placed under the nose) and painful tail pinches (manually applied). Each of the 4 recordings tested with olfactory stimulation exhibited an excitatory response, but a subsequent inhibitory response was difficult to identify (perhaps due to less abrupt and more prolonged stimulus administration). Tail pinches elicited a strong response in all 4 recordings tested, yielding a biphasic (excitatory-inhibitory) pattern of discharge similar to that characteristic of responses to non-noxious auditory, visual, and somatosensory stimuli.

In contrast to the biphasic response pattern typically evoked by sensory stimuli in the above studies, gustatory stimulation (during voluntary consumption of a preferred $5 \%$ aqueous glucose solution) elicited only a decrease in NE-LC discharge. These responses were most apparent in cumulative PSTHs as seen in Figures 4 and 6. Quan- titative PSTH analysis yielded the following results: for SUs, the latency of inhibitory response onset $=59.0 \pm$ $22.4 \mathrm{msec}($ mean $\pm \mathrm{SEM})$ and offset latency $=357.0 \pm$ $32.4 \mathrm{msec}(N=4)$; for MU recordings, the latency of inhibition onset $=44.7 \pm 16.9 \mathrm{msec}$ and offset latency $=$ $420.0 \pm 75.4 \mathrm{msec}(N=6)$. Gustatory responses were unrelated to the motor activity involved in licking, since there was no consistent pattern of NE-LC activity in PSTHs which were triggered at the onset of every lick in an episode (typically 6 to 7 licks/sec for about 2 to $3 \mathrm{sec}$ ); consistent NE-LC response occurred only in PSTHs which were synchronized with the first lick in each trial, i.e., the first lick only for each drop of solution.

Sensory response magnitudes. In our initial studies, NE-LC responses appeared to habituate and dishabituate in rapid succession. Typically, the first 5 to 10 
A

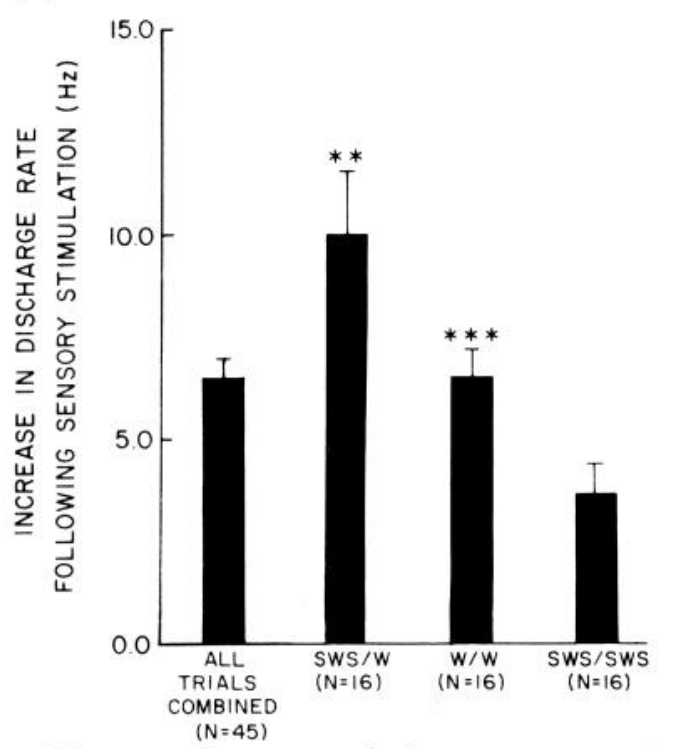

B

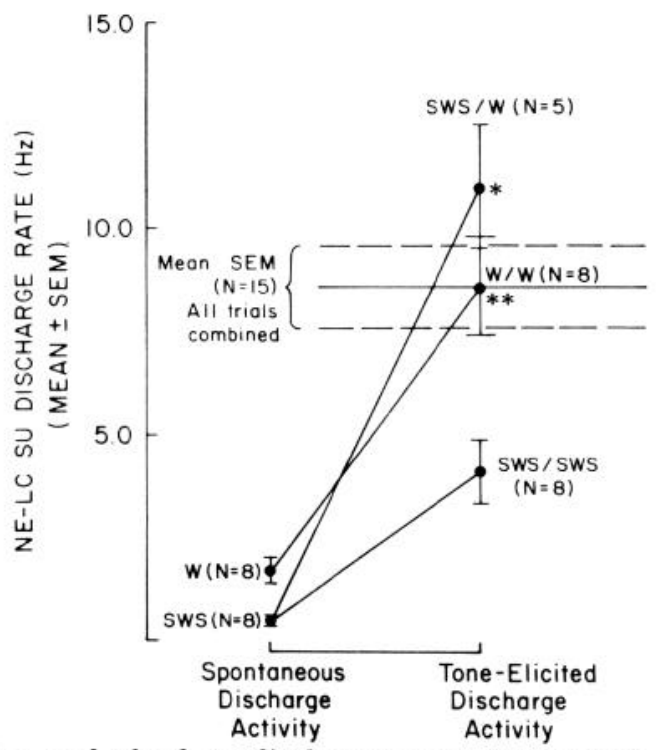

Figure 9. Sensory-evoked response magnitudes and absolute discharge rates for NE-LC neurons as functions of cortical arousal. $A$, Mean response magnitude for SWS/W trials $>$ W/W trials, ${ }^{* *} p<0.005$; mean W/W magnitude $>$ mean SWS/SWS magnitude, ${ }^{* * *} p<0.0005$; mean SWS/W magnitude $>$ mean magnitude for all trials combined $>$ mean SWS/SWS magnitude, $p<0.0005$ for each; paired $t$ tests were used. $B$, Mean absolute discharge rates for 200-msec epochs immediately following stimuli grouped in arousal categories and mean spontaneous discharge rates for these neurons during tonic W and SWS. The lines connecting data points illustrate relative increases in discharge rates. $\mathrm{SWS} / \mathrm{W}>\mathrm{W} / \mathrm{W},{ }^{*} p<0.05$; W $/ \mathrm{W}>\mathrm{SWS} / \mathrm{SWS}$, ${ }^{* *} p<0.0005$; SWS $/ \mathrm{W}>$ all trials combined $>$ SWS/SWS, $p<0.005$ for each; W $>$ SWS, $p<$ 0.005 ; SWS $/ \mathrm{W}>\mathrm{SWS}$ or W, $p<0.005$ for each; W/W $>\mathrm{W}, p<0.0005$; SWS/SWS $>\mathrm{SWS}, p$ $<0.0005$; paired $t$ tests were used.

stimuli elicited robust responses, while the following set of 5 to 10 stimuli elicited very little or no response, followed by pronounced responses to the next few stimuli, etc. (see Figs. 3, 5, and 8). Upon closer examination, however, we found that these fluctuations in response corresponded to changes in the animal's level of vigilance. As illustrated in Figure 8, the magnitudes of response to tone pips were correlated directly with EEG arousal. For quantitative analysis, each stimulus trial was placed in one of three categories according to the S-WC stages immediately preceding and following stimulus presentation, and response magnitude was calculated for each trial (as described under "Materials and Methods"). As shown in Figure 9, response magnitudes for tone pips presented during uninterrupted waking $(\mathrm{W} / \mathrm{W})$ were significantly greater than for those presented during uninterrupted SWS (SWS/SWS) $(p<0.0005$ by paired $t$ test, $N=16$ cells). Furthermore, the largest response magnitudes occurred for tone pips in the SWS/W category, i.e., for stimuli that awakened the animal (SWS/W $>\mathrm{W} / \mathrm{W}$, $p<0.005$ by paired $t$ test, $N=13$ cells). The mean difference $(D)$ between the absolute discharge rates immediately following stimuli in the SWS/W versus $\mathrm{W} / \mathrm{W}$ category $(D=1.21 ; \mathrm{SWS} / \mathrm{W}$ rate $>\mathrm{W} / \mathrm{W}$ rate, $p<0.05$ by paired $t$ tests) was not as pronounced or confident as the corresponding difference between response magnitudes $(D=3.05)$. Conversely, the difference between response magnitudes in the W/W versus SWS/SWS categories $(D=3.05)$ was smaller than the difference between corresponding absolute discharge rates $(D=$
4.50). Responses to flash stimuli generally were not sufficient in magnitude to permit quantitative single trial analysis, but similar general results were observed. Mild auditory, visual, or somatosensory stimuli presented during uninterrupted paradoxical sleep (PS) elicited no response in the 5 NE-LC recordings examined. No habituation of NE-LC response was observed (over 25 to 100 trials) independent of such changes in the level of vigilance.

Sensory-evoked NE-LC responsiveness, as well as spontaneous discharge (Aston-Jones and Bloom, 1981), was observed to decrease during grooming and sweet water consumption, similar to results obtained for sleep (above). Furthermore, these three behavioral states also were correlated with reduced orienting behavior in response to sensory stimuli. However, any sensory stimulus that successfully disrupted such ongoing behavior elicited a robust response in NE-LC activity (qualitative observations). Thus, tonically reduced NE-LC activity accompanied behavioral states characterized by low levels of vigilance, whereas phasic, intense NE-LC discharge corresponded to abrupt increases in vigilance.

Sensory-evoked field potentials. FPs were evoked in the NE-LC by the same sensory stimuli used in the above unit studies, yielding waveforms similar to those occurring spontaneously. As illustrated in Figures 10 through 13, sensory-evoked FPs (or averaged evoked potentials, AEPs) were synchronized temporally with unit responses simultaneously recorded from the same electrodes (see "Materials and Methods"). A negative FP wave accom- 


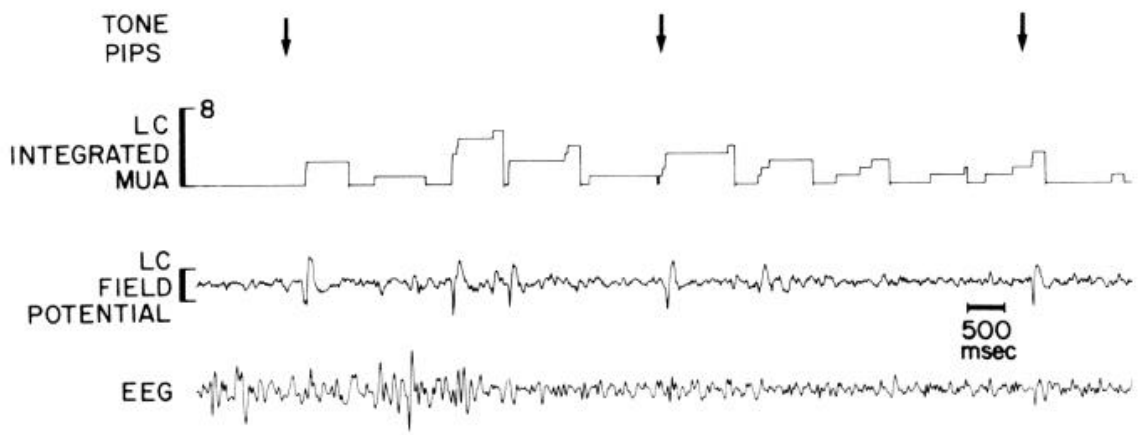

Figure 10. Sensory-evoked FPs and synchronous unit responses in the NE-LC. Tone pips (at arrows) evoke MU and FP responses synchronously. Similar FP waveforms and synchronous MU bursts spontaneously occur in anticipation of W from SWS (between first and second stimuli). Differential recordings between adjacent microwires are separated into FP and discharge traces as described under "Materials and Methods." FP calibration $=100 \mu \mathrm{V}$. MUA, MU activity.

RAT 114
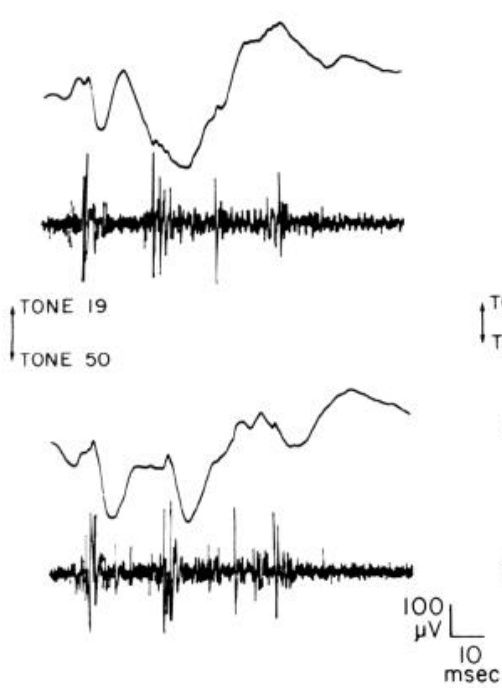

TONE 43

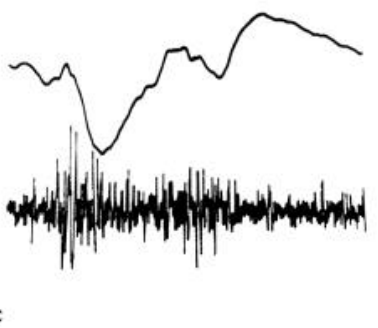

Figure 11. Analog sensory-evoked NE-LC unit responses and FPs. Single oscilloscope sweeps for 2 tone pip trials for each of 2 NE-LC recordings are shown. Differential recordings are separated into FP and unit traces from the same electrodes as described under "Materials and Methods." Tone pip onsets are indicated by arrows. Apparent lag in FP versus unit response partially reflects phase shifts in FP signal produced by filtering.

panied the onset of excitatory unit responses, closely followed by a positive FP deflection. Sensory-evoked FP magnitudes ranged from 100 to $300 \mu \mathrm{V}$ in the negative component and from 50 to $150 \mu \mathrm{V}$ in the positive component.

As found for unit responses (above), the magnitudes of sensory-evoked FPs fluctuated with changes in vigilance. FPs in the SWS/W category were apparently larger than those in the $\mathrm{W} / \mathrm{W}$ category which, in turn, were generally larger than FPs in the SWS/SWS category (illustrated in Figs. 8 and 14). In contrast to unit activity, however, FPs continued to be evoked in the NE-LC during PS (Fig. 15) although with smaller amplitudes than those typically elicited during other S-WC stages.
Topographical specificity. The NE-LC was divided histologically into quadrants (Aston-Jones and Bloom, 1981) to test for possible topographical segregation of unit response properties. There were no differences in Table I properties for SU or MU recordings in different quadrants. Similarly, one-way analyses of variance for response latencies across quadrants yielded no significant differences (for SUs, $p>0.05, N=5,4,4$, and 4 for tones and $3,3,3$, and 2 for flashes in the dorsoanterior (DA), ventroanterior (VA), dorsoposterior (DP), and ventroposterior (VP) quadrants, respectively; for MUs, $p>0.05$, $N=7,3,3$, and 2 for tone pips and $5,0,3$, and 1 for flashes in the DA, VA, DP, and VP quadrants, respectively).

However, when comparing quadrants for response magnitudes, some significant differences emerged. Only DP neurons exhibited SWS/W magnitudes that were significantly greater than those in the $\mathrm{W} / \mathrm{W}$ category $(p$ $<0.05$ by paired $t$ test, $N=3$ cells; $p>0.1$ by paired $t$ tests in the DA and VA quadrants, $N=7$ and 2 cells, respectively; the VP quadrant was not analyzed as there was only 1 cell common to these categories). Also, response magnitudes for DP neurons were significantly greater in the W/W than in the SWS/SWS category at the $p<0.01$ level (by paired $t$ test, $N=4$ cells), while cells in the VA quadrant yielded a similar difference at the $p<0.05$ level only (by paired $t$ test, $N=4$ cells), and DA cells exhibited no significant difference ( $p>0.1$ by paired $t$ test, $N=7$ cells); the VP quadrant was not analyzed (as $N=1$ cell common to these categories). One-way analyses of variance revealed a significant effect of quadrant for response magnitudes in the W/W category only ( $p<0.02, N=16$ cells). Subsequent $t$ tests revealed that DP response magnitudes in the $\mathrm{W} / \mathrm{W}$ category were significantly greater than those for cells in the VA quadrant at the $p<0.005$ level $(N=4$ cells in each quadrant), while neurons in the DA quadrant $(N=7$ cells) yielded significantly higher $\mathrm{W} / \mathrm{W}$ magnitudes than those in the VA quadrant at the $p<0.05$ level only; the VP quadrant was not analyzed (as $N=1$ cell for this category). Thus, cells in the DP quadrant tended to exhibit larger responses to tone pips during $\mathrm{W}$ than other NE-LC neurons. This also appeared to be true for flash 


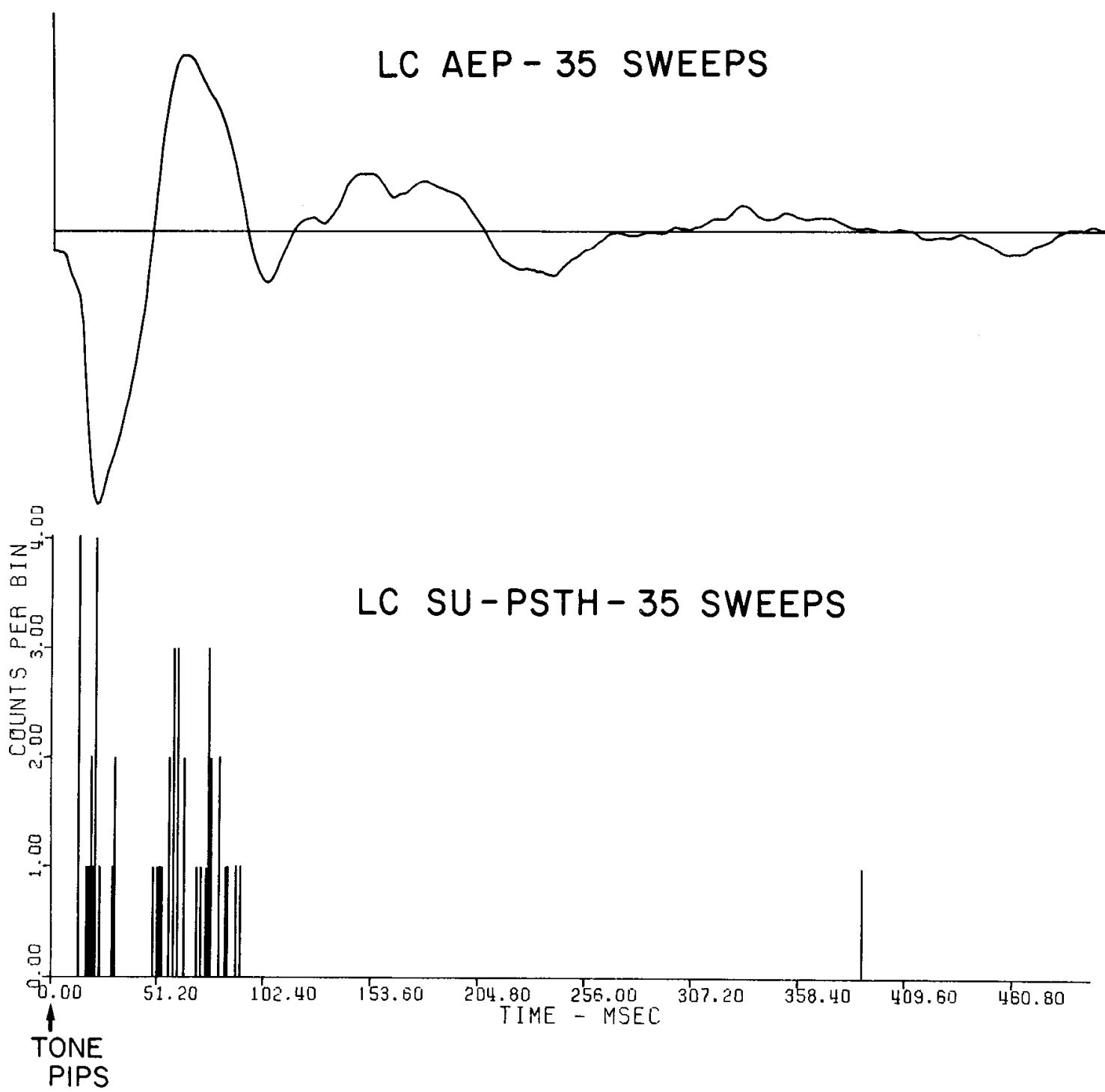

Figure 12. Sensory averaged evoked potential $(A E P)$ and corresponding SU PSTH for one NE-LC recording site. Differential recordings are separated into FP and unit traces from the same electrodes as described under "Materials and Methods." Both records were accumulated for the same tone pip trials (onsets at arrow). The PSTH time axis serves for both records. PSTH bin width $=1 \mathrm{msec}$.

responses, but quantitative comparison across quadrants was precluded by an insufficient number of cells amenable to single trial analysis; however, of the 3 cells with response magnitudes permitting that analysis, 2 were in the DP quadrant.

During the course of these studies, we noted that anomalous activity was exhibited often by NE-LC neurons situated near an edge of the nucleus. We compared our results for neurons located within about $50 \mu \mathrm{m}$ on either side of NE-LC boundaries (edge cells) with results for neurons located more centrally. Thirteen of the 14 exceptions in Table I SU properties were from edge cells as were all 3 exceptions in MU properties. However, PSTHs for SU and MU edge recordings yielded response latencies similar to those for non-edge recordings $(p>$ 0.1 by $t$ tests; $N=5$ edge and 12 non-edge SUs for tone pips, $N=2$ edge and 8 non-edge SUs for flashes; $N=2$ edge and 13 non-edge MUs for tone pips; there were insufficient cases to compare inhibitory flash response latencies or MU excitatory flash response latencies).
Qualitative data collected on 91 non-LC pontine SUs during the course of these studies are summarized in Table III. Comparing Table I with Table III reveals that a relatively small percentage of non-LC pontine neurons exhibited discharge properties that were characteristic of NE-LC neurons (percentages differ between corresponding properties in Table I and Table III at $p<0.001$ by $\chi^{2}$ tests for two independent samples). There were too few non-LC neurons quantitatively examined to compare their latencies or durations with NE-LC neurons confidently.

\section{Discussion}

The present results expand the set of characteristic properties for NE-LC activity in unanesthetized behaving rats (Aston-Jones and Bloom, 1981). These neurons exhibit prompt, biphasic responses to non-noxious auditory, visual, and somatosensory stimuli. Such responses, consisting of a short burst of impulses followed by a prolonged silence, typically are exhibited for each stim- 


\section{LC AEP - 50 SWEEPS}

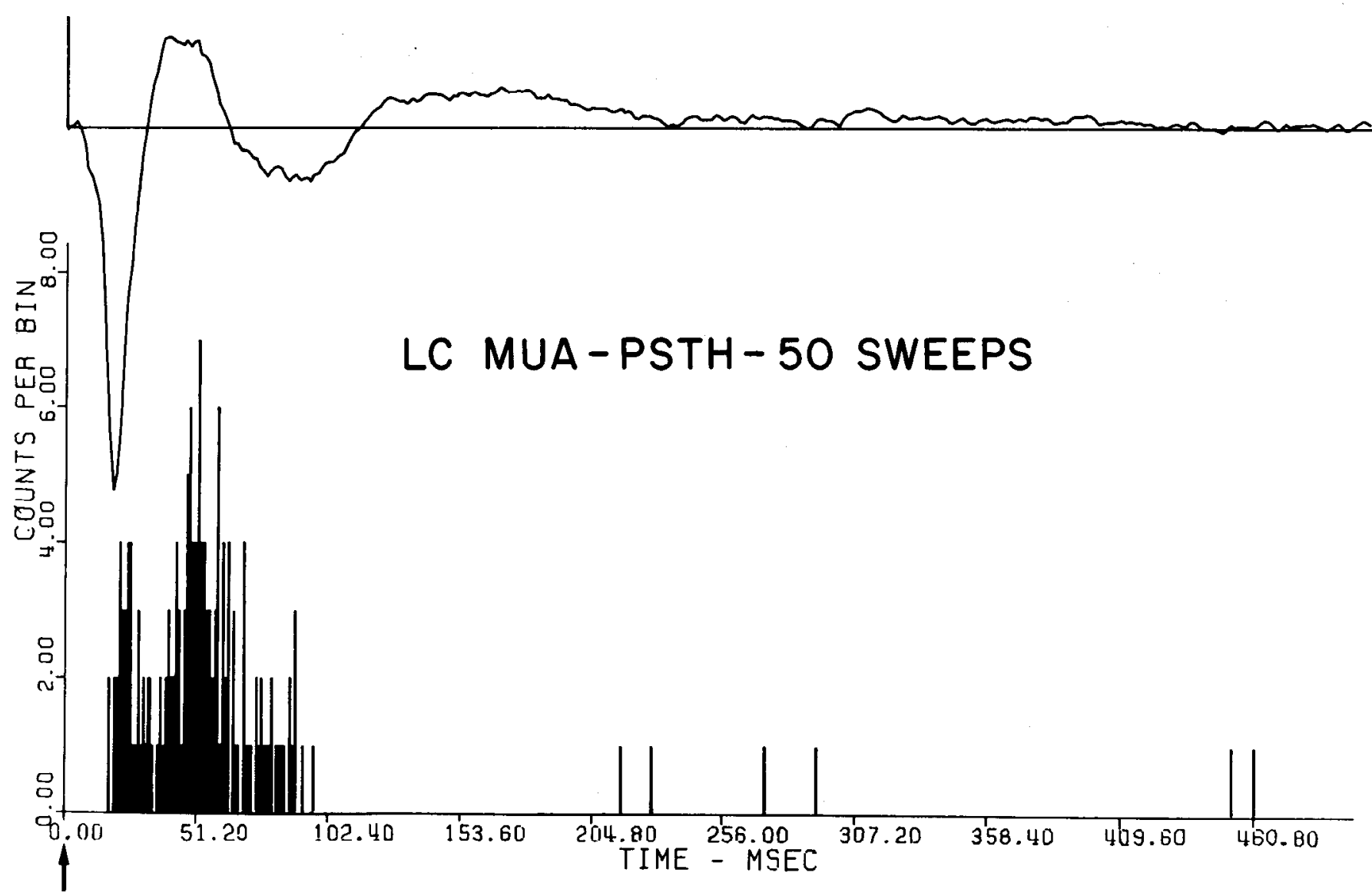

TONE

PIPS

Figure 13. Sensory averaged evoked potential $(A E P)$ and corresponding MU PSTH for one NE-LC recording site. Recordings were obtained and data are illustrated as described for Figure 12.

\section{LC TONE AEP'S}

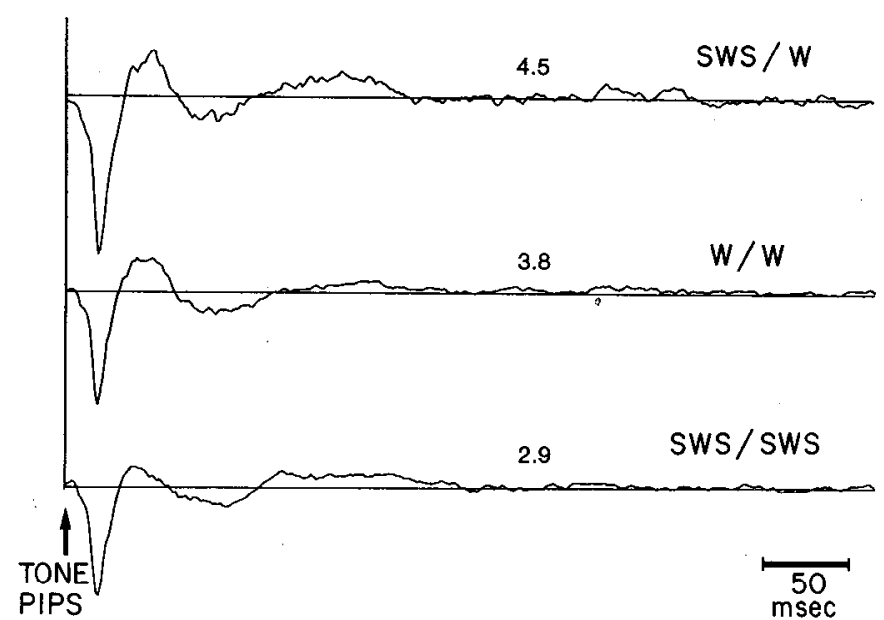

Figure 14. Sensory averaged evoked potential $(A E P) \mathrm{mag}$ nitudes in NE-LC as a function of cortical arousal. Three AEPs generated from a single series of consecutive tone pip trials are segregated by cortical arousal categories. Magnitudes were normalized for number of trials. Above each AEP is the corresponding mean number of MU impulses (from the same electrodes) per trial. Differential recordings, 0.1- to $150-\mathrm{Hz}$ bandpass filtering for FP trace.

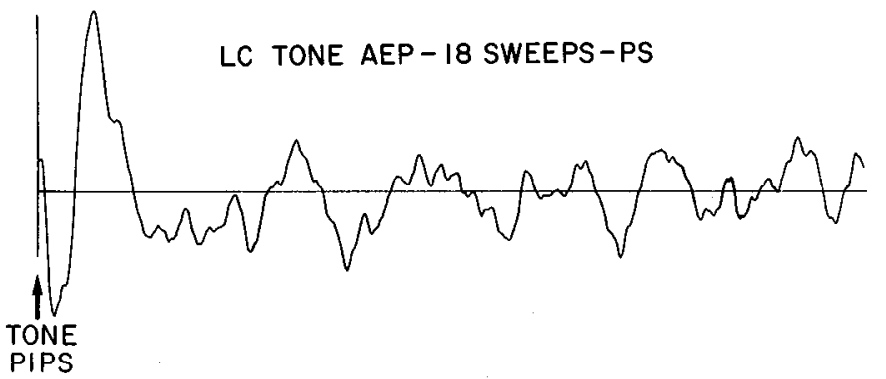

NON-SYNC AEP-PS

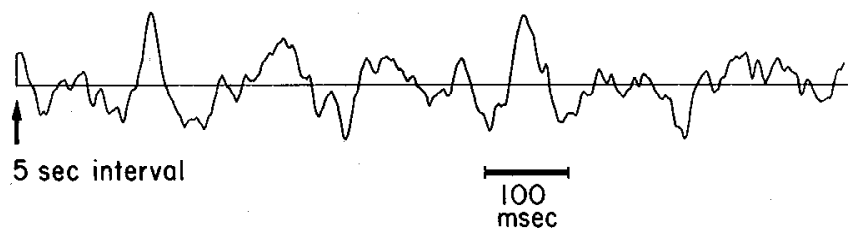

Figure 15. Sensory averaged evoked potential ( $A E P$ ) in the NE-LC during PS. Sweeps incorporated in the upper trace were triggered at the tone pip onsets (arrows; 4-sec interval). The lower trace was generated from the same PS recording epoch (on tape), but the sweeps were triggered at regular 5 -sec intervals, independent of stimulus presentations. Differential recordings, $0.1-$ to $150-\mathrm{Hz}$ bandpass filtering. 
TABLE III

Proportions of non-LC pontine SU recordings with discharge properties characteristic of NE-LC neurons

The format is the same as in Table I. Properties in the columns apply to all pontine recording locations.

\begin{tabular}{|c|c|c|c|c|c|}
\hline Recording Location & $\begin{array}{c}\text { Slow, Tonic } \\
\text { Spontaneous Rate }\end{array}$ & $\begin{array}{l}\text { Initial + } \\
\text { Auditory }\end{array}$ & $\begin{array}{c}\text { Initial }+ \\
\text { Visual }\end{array}$ & $\begin{array}{c}\text { Initial + } \\
\text { Somatesthetic }\end{array}$ & $\begin{array}{c}\text { Biphasic }(+,-) \\
\text { Responses for at } \\
\text { least Two Modalities }\end{array}$ \\
\hline \multicolumn{6}{|l|}{50 to $250 \mu \mathrm{m}$ from LC } \\
\hline Parabrachial nuclei & $1 / 6$ & $1 / 5$ & $1 / 2$ & $0 / 2$ & $1 / 2$ \\
\hline Mesencephalic reticular formation & $0 / 2$ & & & & \\
\hline Pontine reticular formation & $3 / 10$ & $3 / 5$ & $1 / 3$ & $1 / 4$ & $1 / 3$ \\
\hline Mesencephalic nucleus $\mathrm{V}^{a}$ & $0 / 4$ & $2 / 3$ & $1 / 1$ & $3 / 3$ & $0 / 3$ \\
\hline Central gray & $1 / 8$ & $3 / 7$ & $2 / 4$ & $1 / 4$ & $1 / 3$ \\
\hline \multicolumn{6}{|l|}{300 to $3000 \mu \mathrm{m}$ from $\mathrm{LC}$} \\
\hline Parabrachial nuclei & $1 / 2$ & $0 / 1$ & $0 / 1$ & $0 / 2$ & \\
\hline Mesencephalic reticular formation & $3 / 19$ & $2 / 9$ & $2 / 7$ & $4 / 12$ & $1 / 3$ \\
\hline Pontine reticular formation & $2 / 28$ & $7 / 15$ & $2 / 12$ & $2 / 12$ & $0 / 6$ \\
\hline Mesencephalic nucleus $\mathrm{V}^{a}$ & $2 / 5$ & $1 / 3$ & $0 / 2$ & $1 / 2$ & $1 / 1$ \\
\hline Central gray & $1 / 7$ & $5 / 5$ & $1 / 1$ & $2 / 2$ & $1 / 5$ \\
\hline
\end{tabular}

${ }^{a}$ Nucleus of the fifth cranial nerve.

ulus modality in individual NE-LC recordings. In contrast, these neurons exhibit only inhibitory responses to voluntarily consumed, preferred gustatory stimuli. Thus, NE-LC discharge is characterized by polysensory responsiveness, yielding two modality-specific patterns of evoked activity.

Tone pip response latencies were 30 to 50 msec shorter than for flash stimuli; this difference may be attributable to retinal transmission time, estimated at $30 \mathrm{msec}$ in cat (Creutzfeldt, 1970). These auditory response latencies approximate the latencies reported for similar responses to electrical stimulation of certain peripheral nerves (Aghajanian et al., 1977; Takigawa and Mogenson, 1977).

Excitatory response magnitudes fluctuated during trains of stimuli in the present study, which might be interpreted as habituation and dishabituation in the NELC. However, these fluctuations were associated systematically with simultaneous changes in the level of vigilance so that the largest responses occurred for stimuli that awakened rats and the smallest were elicited by identical stimuli during uninterrupted sleep. Although habituation of NE-LC responsiveness may eventually occur dissociated from vigilance changes, none was observed here with up to 100 consecutive stimuli. Sensory response magnitudes also were apparently reduced for certain behaviors within active waking, i.e., during grooming or consumption of sweet water, times when behavioral orienting responses were suppressed. However, stimuli that successfully interrupted either of these behaviors elicited robust responses in NE-LC neurons. Thus, sensory-evoked activity in the NE-LC, like spontaneous discharge (Aston-Jones and Bloom, 1981), is suppressed tonically during sleep, grooming, and consumption but is phasically enhanced when such ongoing behavior is disrupted, corresponding to a change in behavioral state.

Mild sensory stimuli also evoked FPs in the NE-LC, eliciting waveforms closely resembling those occurring spontaneously. Similar events have been observed recently in the LC area by Kaufman and Morrison (1981) in unanesthetized behaving rats, but their use of large diameter electrodes makes it difficult to specify sites which generate recorded signals. The present study, how- ever, provides more definitive evidence that FPs do arise from NE-LC activity: (1) FPs were recorded from sites discretely localized to the NE-LC. (2) FPs were obtained with monopolar etched tungsten microelectrodes as well as with differential pairs of adjacent microwire electrodes. Both of these recording techniques probably monitor activity from a relatively small volume of tissue only. (3) FPs were synchronized temporally with unit activity simultaneously recorded from the same electrodes. In particular, the negative FP component typically was accompanied by a burst of unit impulses, as expected for neurons that generate the corresponding FP current sink (Steriade and Hobson, 1976). (4) NE-LC neurons were generally homogeneous in their discharge properties, and neurons in MU populations were synchronized markedly during bursts of impulses. Such concerted activity in a tightly packed group of cells fulfills theoretical requirements (Steriade and Hobson, 1976) for sites generating FPs and implies that NE-LC neurons may function as a homogeneous ensemble.

The relationship between sensory response magnitudes in the NE-LC and vigilance levels implies that there are two significant, distinct influences on NE-LC activily: (1) excitatory inputs mediating sensory-evoked discharge and (2) inhibitory systems that modulate NELC excitability according to vigilance or behavioral state. The present FP data offer additional insight as to the factors controlling NE-LC activity. Spontaneous and sensory-evoked FPs without unit activity during PS may reflect concerted excitatory postsynaptic potentials in the presence of strong, tonic inhibition which prevents discharge. Phasic, excitatory inputs concurrent with tonic, inhibitory inputs resemble factors known to be operating on motoneurons during PS (Chase, 1980), where impulse generation is prevented despite intense excitatory barrages. This would be consistent with previous proposals (McCarley and Hobson, 1975) that NELC discharge is incompatible with PS and would suggest that suppression of these neurons plays a critical role in PS episodes.

Future experiments are planned to determine if varying intensities of excitatory and inhibitory inputs to NELC neurons similarly underlie certain present results for 
W and SWS as well. Increased inhibition during SWS, grooming, and consumption could produce the corresponding decreases observed in spontaneous and sensoryevoked impulse activities. Thus, the NE-LC may integrate CNS signals that reflect external sensory events with those that convey internal vegetative requirements. The relative intensities of activity in these two afferent systems could determine the level of NE-LC discharge which then may influence the global orientation of brain and behavioral activities.

Although some sensory response characteristics of NELC neurons in unanesthetized behaving rats resemble results reported for anesthetized rats, many fundamental properties differ markedly. Previous studies (Graham and Aghajanian, 1971; Bunney et al., 1975; Cedarbaum and Aghajanian, 1976; Aghajanian et al., 1977; Bird and Kuhar, 1977; Aghajanian, 1978), as well as our own observations, indicate that these cells in anesthetized animals respond only to strong, noxious environmental stimuli. In contrast, we found that mild, non-noxious sensory stimuli of many modalities elicit pronounced NE-LC responses in unanesthetized behaving preparations. In our unanesthetized preparation, pronounced lability was observed in spontaneous NE-LC discharge with MU recordings, apparently resulting from responses to background environmental stimuli. (Such bursty discharge was less apparent in spontaneous SU activity, probably due to the smaller sampling of impulse activity.) In fact, increased sensory responsivity is the most prominent difference between behaving and anesthetized rats' NELC discharge. Reduced responsivity under anesthesia may correspond to the relationship between response amplitude and vigilance found in the present study. By maintaining a low vigilance level, anesthesia may decrease NE-LC sensory responsiveness much like sleep does in unanesthetized rats. The ineffectiveness of most sensory stimuli in anesthetized rats has led some investigators to propose that the NE-LC system is involved primarily in nociception, fear, or anxiety (Lader, 1974; Gray et al., 1975; Redmond and Huang, 1979). The present results indicate a much broader range of environmental influences on NE-LC discharge, and therefore, a more general role for this system in brain and behavioral activity.

\section{Summary and Hypothesis}

The studies described here and in the preceding (Aston-Jones and Bloom, 1981) paper have demonstrated the following set of characteristic properties for NE-LC neurons. (1) Spontaneous discharge co-varies with stages of the S-WC, exhibiting rates directly related to the level of vigilance. (2) Spontaneous discharge fluctuates with and anticipates phasic cortical epochs (spindle activity during SWS) as well as tonic cortical periods (S-WC stages, except W after PS). (3) Discharge is not apparently linked to specific movements but does correspond to orienting, grooming, and consumption behaviors. (1) FPs occur spontaneously in the NE-LC and are synchronized temporally with unit activity (during W and SWS) simultaneously recorded from the same electrodes. (5) Biphasic FPs and synchronous unit responses are evoked by mild, non-noxious environmental stimuli of many modalities. (6) Spontaneous and sensory-evoked FPs persisted during PS in the virtual absence of unit activity. (7) Spontaneous as well as sensory-evoked activity is reduced tonically during sleep, grooming, and consumption but is enhanced phasically when such behaviors are interrupted. (8) SU and MU recordings throughout the NE-LC yield homogeneous discharge properties.

Our present results, together with previous data on the postsynaptic effects of NE (Foote et al., 1975; Segal and Bloom, 1976a, b; Freedman et al., 1977), lead us to reevaluate proposals of NE-LC function and to offer a new working hypothesis for the role of this system in brain and behavioral activity. A global release of NE (e.g., following environmental stimulation that elicits robust NE-LC discharge) may enhance signals in brain systems engaged by exogenous sensory stimuli and simultaneously suppress CNS activity associated with tonic vegetative functions which are low in priority for phasic adaptive behavior. Sleep, grooming, and consumption, as endogenously generated repetitive behaviors, may critically depend upon low levels of NE-LC impulse activity: vigorous discharge may disrupt or disengage such internally oriented behavioral patterns by enhancing signalto-noise characteristics (and, therefore, transmission flow) in CNS pathways important for appropriate response to unexpected external events. We propose, therefore, that the NE-LC system may function to facilitate transitions between behavioral states. By selectively augmenting CNS activity engaged by rapidly changing exogenous stimuli, robust NE-LC discharge may bias the global orientation of behavior toward coping with phasically imperative events in the external environment.

\section{References}

Aghajanian, G. (1978) Tolerance of locus coeruleus neurons to morphine and suppression of withdrawal response by clonidine. Nature 276: 186-188.

Aghajanian, G., J. Cedarbaum, and R. Wang (1977) Evidence for norepinephrine-mediated collateral inhibition of locus couruleus neurons. Brain Res. 136: 570-577.

Amaral, D., and H. Sinnamon (1977) The locus coeruleus: Neurobiology of a central noradrenergic nucleus. Prog. Neurobiol. 9: 147-196.

Aston-Jones, G., and F. E. Bloom (1981) Activity of norepinephrine-containing locus coeruleus neurons in behaving rats anticipates fluctuations in the sleep-waking cycle. J. Neurosci. 1: 876-886.

Aston-Jones, G., M. Segal, and F. E. Bloom (1980) Brain aminergic axons exhibit marked variability in conduction velocity. Brain Res. 195: 215-222

Bird, S., and M. Kuhar (1977) Iontophoretic application of opiates to the locus coeruleus. Brain Res. 122: 523-533.

Bunney, B., J. Walters, M. Kuhar, R. Roth, and G. Aghajanian (1975) D- and L-amphetamine stereoisomers: Comparative potencies in affecting the firing of central dopaminergic and noradrenergic neurons. Psychopharmacol. Commun. 1: 177.

Cedarbaum, J., and G. Aghajanian (1976) Noradrenergic neurons of the locus coeruleus: Inhibition by epinephrine and activation by the $\alpha$-antagonist piperoxane. Brain Res. 112: 413-419.

Chase, M. (1980) The motor functions of the reticular formation are multifaceted and state-determined. In The Reticular System Revisited, J. A. IIobson and M. Brazier, eds., pp. 449472, Raven Press, New York.

Clark, T. (1979) The locus coeruleus in behavioral regulation Evidence for behavior-specific versus general involvement. Behav. Neural Biol. 25: 271-300. 
Creutzfeldt, O. (1970) Some principles of synaptic organization in the visual system. In The Neurosciences: Second Study Program, F. Schmitt, ed., pp. 630-647, Rockefeller University Press, New York.

Foote, S. L., R. Freedman, and A. P. Oliver (1975) Effects of putative neurotransmitters on neuronal activity in monkey auditory cortex. Brain Res. 86: 229-242.

Foote, S., G. Aston-Jones, and F. E. Bloom (1980) Impulse activity of locus coeruleus neurons in awake rats and squirrel monkeys is a function of sensory stimulation and arousal. Proc. Natl. Acad. Sci. U. S. A. 77: 3033-3037.

Freedman, R., B. Hoffer, D. Woodward, and D. Puro (1977) Interaction of norepinephrine with cerebellar activity evoked by mossy and climbing fibers. Exp. Neurol. 55: 269-288.

Graham, A., and G. Aghajanian (1971) Effects of amphetamine on single cell activity in a catecholamine nucleus, the locus coeruleus. Nature 234: 100.

Gray, J., N. McNaughton, D. James, and P. Kelley (1975) Effect of minor tranquilizers on hippocampal theta rhythm mim. icked by depletion of forebrain noradrenaline. Nature 258: 424-425.

Jones, G., M. Segal, S. Foote, and F. E. Bloom (1979) Locus coeruleus neurons in freely moving rats exhibit pronounced alterations of firing rate during sensory stimulation and stages of the sleep-wake cycle. In Catecholamines: Basic and Clinical Frontiers, E. Usdin, I. Kopin, and J. Barchas, eds., pp. 643-645, Pergamon Press, New York.
Kaufman, L., and A. Morrison (1981) Spontaneous and elicited PGO spikes in rats. Brain Res. 214: 61-72.

Lader, M. (1974) The peripheral and central role of the catecholamines in the mechanisms of anxiety. Int. Pharmacopsychiatry 9: 125-137.

McCarley, R., and J. Hobson (1975) Neuronal excitability modulation over the sleep cycle: A structural and mathematical model. Science 189: 58-60.

Ramm, P. (1979) The locus coeruleus, catecholamines, and REM sleep: A critical review. Behav. Neural Biol. 25: 415448 .

Redmond, D., and Y. Huang (1979) New evidence for a locus coeruleus-norepinephrine connection with anxiety. Life Sci. 25: 2149-2162.

Segal, M., and F. E. Bloom (1976a) The action of norepinephrine in the rat hippocampus: III. Hippocampal cellular responses to locus coeruleus stimulation in the awake rat. Brain Res. 107: 499-511.

Segal, M., and F. E. Bloom (1976b) The action of norepinephrine in the rat hippocampus: IV. The effects of locus coeruleus stimulation on evoked hippocampal unit activity. Brain Res. 107: 513-525.

Steriade, M., and J. A. Hobson (1976) Neuronal activity during the sleep-waking cycle. Prog. Neurobiol. 6: 155-376.

Takigawa, M., and G. Mogenson (1977) A study of inputs to antidromically identified neurons of the locus coeruleus. Brain Res. 135: 217-230. 